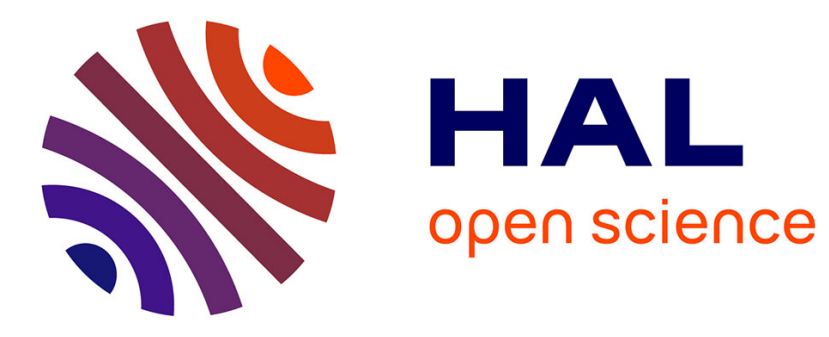

\title{
Automatic Bifurcation Detection in Coronary X-Ray Angiographies
}

Asma Kerkeni, Asma Ben Abdallah, Antoine Manzanera, Ibtihel Nouira, Mohamed Hedi Bedoui

\section{- To cite this version:}

Asma Kerkeni, Asma Ben Abdallah, Antoine Manzanera, Ibtihel Nouira, Mohamed Hedi Bedoui. Automatic Bifurcation Detection in Coronary X-Ray Angiographies. 13th International Conference Computer Graphics, Imaging and Visualization (CGIV 2016), 2016, Beni Mellal, Morocco. pp.333 338, 10.1109/CGiV.2016.70 . hal-01451154

\section{HAL Id: hal-01451154 https://hal.science/hal-01451154}

Submitted on 31 Jan 2017

HAL is a multi-disciplinary open access archive for the deposit and dissemination of scientific research documents, whether they are published or not. The documents may come from teaching and research institutions in France or abroad, or from public or private research centers.
L'archive ouverte pluridisciplinaire HAL, est destinée au dépôt et à la diffusion de documents scientifiques de niveau recherche, publiés ou non, émanant des établissements d'enseignement et de recherche français ou étrangers, des laboratoires publics ou privés. 


\title{
Automatic Bifurcation Detection in Coronary X-Ray Angiographies
}

\author{
Asma Kerkeni*, Asma Ben Abdallah*, Antoine Manzanera ${ }^{\ddagger}$, Ibtihel Nouira* and Mohamed Hedi Bedoui* \\ *Laboratoire Technologie et Imagerie Médicale, \\ Faculté de Médecine, Université de Monastir, Tunisie \\ Email: asma.kerkeni@gmail.com,asma.babdallah@cristal.rnu.tn, ibtihelnouira@gmail.com,medHedi.Bedoui@fmm.rnu.tn \\ ¥Unité d'Informatique et d'Ingénierie des Systèmes, \\ ENSTA-ParisTech, Université de Paris-Saclay, France \\ Email: antoine.manzanera@ensta-paristech.fr
}

\begin{abstract}
The detection of vascular bifurcation in X-ray images is important for several medical applications. They are used as landmarks for image registration, vessel segmentation and tracking. Although many bifurcation extraction methods have been proposed in recent years, very few work deals with coronary bifurcation in X-ray images. In this paper, we present a new bifurcation detector based on the multiscale Hessian analysis. It can be seen as a scale specific Histogram of Eigenvectors weighted by the vesselness measure. Pixels with three peaks in their immediate neighbourhood are considered as bifurcation candidates. Based on this detector, a novel bifurcationness measure is proposed. The method is tested on real coronary artery angiographies and shows better results compared to other bifurcation detectors.
\end{abstract}

Keywords-hessian, eigenvector, vesselness, histogram, bifurcationness.

\section{INTRODUCTION}

Cardiovascular diseases are the leading cause of deaths all over the world and therefore, researches in modern medical image processing aim at developing reliable and robust medical tools to assist clinicians in data analysis and assistance during diagnostic and interventional procedures. Nowadays, X-ray Coronary Angiography (XCA) is the gold standard for the assessment of cardiovascular diseases (CVD). The angiograms obtained by the XCA enable to reveal the initial CVD symptoms by the morphological features of the coronary arteries such as diameter, length, branching angle, and tortuosity.

The detection of vascular bifurcations in X-ray images is particularly important since these branching points can be used as landmarks for image registration and vessel tracking [1]. Although several methods deal with vascular bifurcation detection [2]-[9], little work has been devoted to coronary bifurcations in X-ray images.

In many blood vessel images such as retinal images, branching point detection generally focuses on binary images. Thus, the original structures are reduced to one-pixel-wide vascular trees through segmentation and thinning. The bifurcations and crossovers are then obtained by counting the pixels local connectivity in the skeleton image [2], [3],
[5]. Such approaches are usually affected by the thinning artifacts like tailing, necking, spurs or staircase artifact [9]. Other methods estimate initial bifurcation candidates by the classic Harris corner detector [10] based on the first image derivatives. However, in coronary angiographic images, the first-derivative information is usually too noisy and many false detections occur.

Hence many detectors rely on the second image derivative, presented by the Hessian matrix, which have strong response on blobs and ridges [11]. General Hessian-based features detectors such as difference of Gaussian (DOG) [12], Hessianaffine [11], speeded up robust features (SURF) [13], smallest Hessian eigenvalue [7], Determinant of Hessian [7] and the Laplacian were proposed for blob detection in the computer vision field. The major limit of such detectors is the large rate of false positives since blob points, high curved vessel location, and even some noise artifacts are often confused with branching points.

In [14] a novel bifurcation detector named Histogram of Eigenvectors (HOE) is proposed by analogy to the Histogram of Gradient (HOG) feature [15]. The HOE is combined with other features in order to define a random forest classifier for bifurcation detection in 3D vascular images [14]. The essential thought behind the HOE is that bifurcation can be detected thanks to the observation of local vessel orientation provided by the eigenvector corresponding to the smaller Hessian eigenvalues. Thus, for each pixel, an HOE is computed in a local neighbourhood and pixels with three peaks are most likely to be branching points.

However, it is unclear how the considered local neighbourhood is selected, though its size is critical since the images often present a wide range of vessel width and a fixed window may lead to underestimate a large bifurcation or combine two nearby vessels in the same window.

In this work, we propose a novel scale specific Histogram of Eigenvector detector, where the local neighbourhood of each pixel is taken as the optimal vesselness scale, yielding a more accurate branching detection and a better bifurcation size estimation. We also propose a novel bifurcationness measure on the basis of this descriptor. 
The remaining part of the paper is organized as follows: in section 2 we present the proposed method by describing the different Hessian features and the HOE calculation steps. The experiments and results are presented in section 3 . Section 4 summarizes our paper and discusses the possible ways for future works.

\section{METHOD}

The overview of our proposed vessel bifurcation detector is shown in Fig.1. First, the eigenvalues and eigenvectors of the Hessian matrix are obtained in multiple scales to provide the structural and directional information. Then, the maximum response with the corresponding optimal scale is retained for each pixel of the image using the Frangi vesselness filter which is built on the Hessian eigenvalues. Finally, for each pixel, the Histogram of Eigenvectors is computed in an appropriate local neighborhood given by the optimal scale of the pixel.

\section{A. Multiscale Hessian Feature extraction}

The idea behind multiscale image analysis is to add a new dimension to the analysis which is the image scale. The image is transformed into a set of blurred images, each representing the original image, but at a different scale [16]. These blurred images are obtained by convolving the initial image $I_{0}(p)=I_{0}(x, y)$ with a Gaussian kernel to represent the information at a certain scale.

$$
I_{\sigma}(p)=I_{\sigma}(x, y)=I_{0}(x, y) \otimes G_{\sigma}(x, y)
$$

where $I_{\sigma}$ is an image of the scale space, $p=(x, y)$ is a pixel location, $\otimes$ represents the convolution operation and $G_{\sigma}(x, y)$ is the 2D Gaussian kernel with standard deviation $\sigma$ defined as:

$$
G_{\sigma}(p)=G_{\sigma}(x, y)=\frac{1}{\sqrt{2 \pi \sigma^{2}}} \exp -\frac{\left(x^{2}+y^{2}\right)}{2 \sigma^{2}}
$$

where $\sigma \in \Sigma=\left\{\sigma_{\min }, . ., \sigma_{\max }\right\}, \sigma_{\min }$ and $\sigma_{\max }$ are set according to the approximate width of the smallest and largest vessel to be detected [16].

The 2-by-2 Hessian matrix at a given pixel is composed of the second-order derivatives of an image intensity function Thus, in the scale space, it can be obtained at each point by Eq.3:

$$
H_{\sigma}(p)=\left(\begin{array}{ll}
\frac{\partial^{2} I_{\sigma}(x, y)}{\partial x^{2}} & \frac{\partial^{2} I_{\sigma}(x, y)}{\partial y \partial x} \\
\frac{\partial^{2} I_{\sigma}(x, y)}{\partial x \partial y} & \frac{\partial^{2} I_{\sigma}(x, y)}{\partial y^{2}}
\end{array}\right)
$$

The Hessian matrix is symmetric, therefore has two real eigenvalues $\lambda_{1}$ and $\lambda_{2}$ and two associated eigenvectors $e_{1}$ and $e_{2}$. The signs as well as the relative and absolute magnitudes of eigenvalues characterize the local shape of the intensity in the image. If we assume that we seek to characterize black vessels on a white background, which is

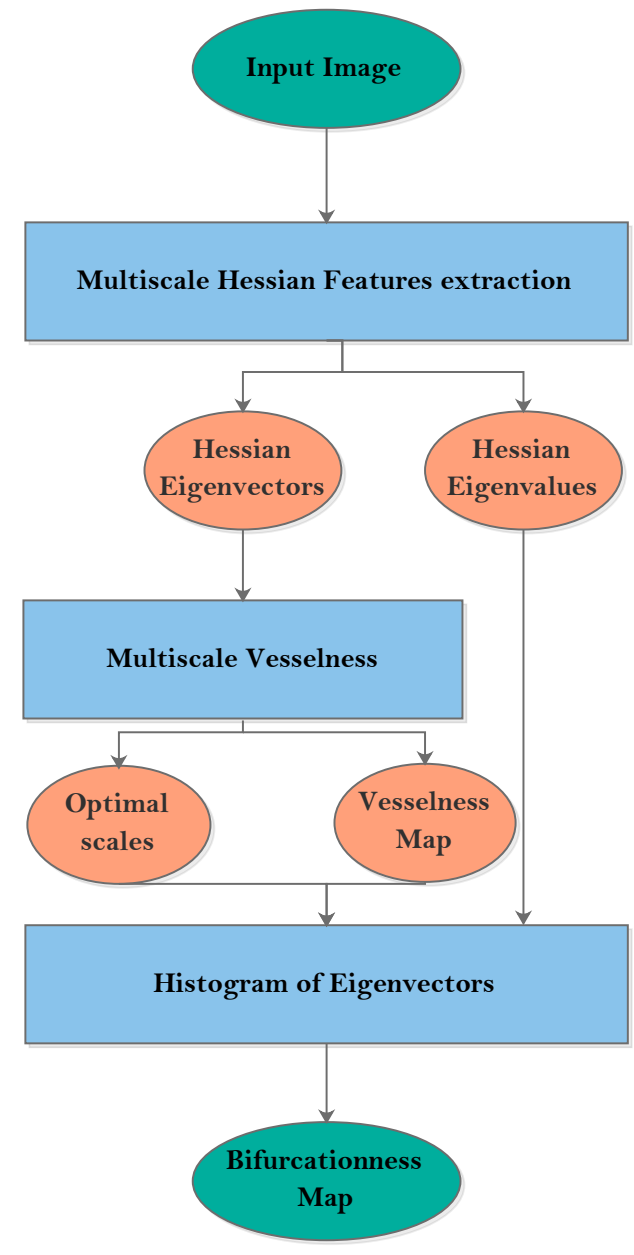

Figure 1. Overview of the multiscale bifurcation detection method.

the case of the angiographic images, a pixel belonging to a vessel region will be given by small (ideally 0) $\lambda_{1}$ and a high positive value of $\lambda_{2}\left(\left|\lambda_{1}\right|<\left|\lambda_{2}\right|\right)$

\section{B. Multiscale Vesselness Filter}

The filter of Frangi [17] is often considered as the current gold-standard due to its simplicity, intuitive formulation and good vascular structure enhancement of medical angiographic images [18], [19]. The thought behind this filter is that the Hessian eigenvalues are geometrically interpreted as principal vessel curvatures. Generally, vessels have strong variation perpendicular to the vessel centreline (high $\lambda_{2}$ ) and weak variation along it (weak $\lambda_{1}$ ). On the basis of these observations, Frangi et al. introduce two measures to describe structures in images: $R_{b}=\frac{\lambda_{1}}{\lambda_{2}}$ is the bloblike structure measure and $S=\|H\|_{F}=\sqrt{\lambda_{1}^{2}+\lambda_{2}^{2}}$, the Frobenius norm of the Hessian matrix, is the second-order structureness measure. These measures were combined in a 


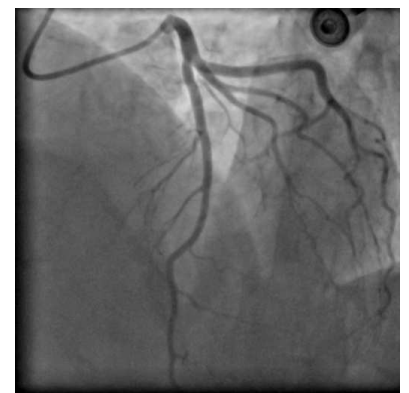

(a)

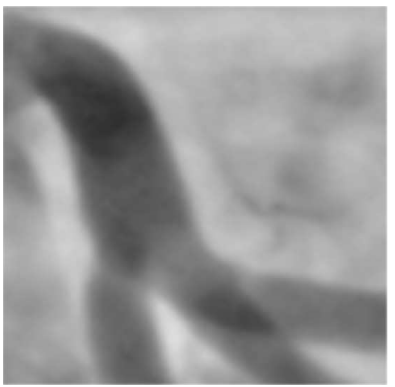

(c)

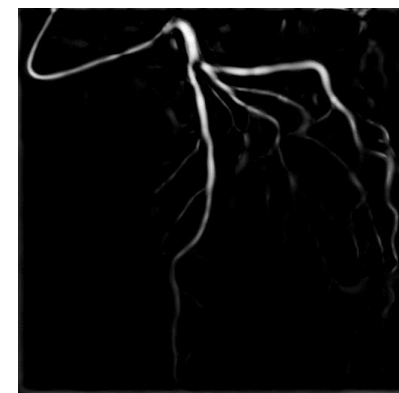

(b)

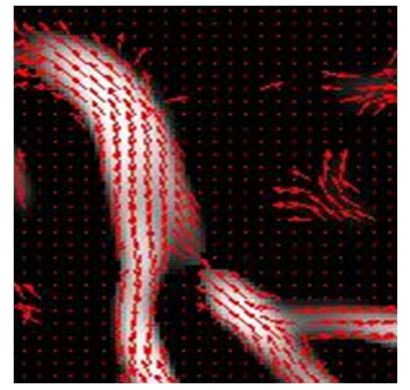

(d)
Figure 2. Hessian-based features: (a) original image; (b) Frangi vesselness response; (c) a small part of the original image; (d) corresponding first eigenvector field superimposed to the vesselness response.

vesselness function as given by Eq.4.

$$
V_{\sigma}(p)= \begin{cases}0 & \text { if } \lambda_{2}<0 \\ \exp \left(-\frac{R_{b}^{2}}{2 \beta^{2}}\right)\left(1-\exp \left(-\frac{S^{2}}{2 c^{2}}\right)\right) & \text { otherwise }\end{cases}
$$

Where parameters $\beta$ and $c$ are thresholds which control the filter's sensitivity to $R_{b}$ and $S$ respectively. According to scale space theory, $V_{\sigma}(p)$ will be maximum up to a normalisation factor when the width of the vessel in pixel $p$ matches a suitable scale factor $\sigma$. Thus, In the multi-scale vessel enhancement algorithm, $V_{\sigma}$ is computed for different scales, then the largest one is taken as the final filter output.

$$
V_{\text {opt }}(p)=\max _{\sigma \in \Sigma} V_{\sigma}(p)
$$

The optimal scale $\sigma^{*}$ corresponding to the maximum of vesselness is given by Eq.6.

$$
\sigma^{*}(p)=\arg \max _{\sigma \in \Sigma} V_{\sigma}(p)
$$

\section{Vessel Direction Information}

The eigenvectors $e_{1}$ and $e_{2}$ of the Hessian matrix characterize the local shape of the intensity. $e_{1}$ gives the direction of lower second derivative, i.e. the direction of a potential linear structure, while $e_{2}$ gives the direction of the stronger second derivative, i.e. the normal direction to a potential linear structure. Thus the proposed direction information $D_{\sigma}$ can be simply given by the following expression:

$$
D_{\sigma}(p)= \begin{cases}0 & \text { if } \lambda_{2}<0 \\ e_{1} & \text { otherwise } .\end{cases}
$$

The final vessel direction is given by the direction $D_{\sigma}$ at the scale $\sigma$ that provided the optimal response of the filter $V_{\text {opt }}(p)$ as given by Eq.8.

$$
D_{\text {opt }}(p)=D_{\sigma^{*}}(p)
$$

Fig. 2 shows the vesselness and direction features of an X-ray angiogram where Fig.2(b) is the vesselness Map obtained by the Frangi filter and Fig.2(d) is the final eigenvector field of the region of interest shown in Fig.2(c).

\section{Histogram of Eigenvectors}

Once the vesselness and local orientation features are computed, the Histogram of Eigenvectors $(\mathrm{HoE})$ is obtained through the following steps:

1) For each pixel $p$, estimate the optimal scale $\sigma^{*}(p)$ using the equation Eq.6.

2) Construct a circular histogram by casting a vote for each direction vector on the circle centred on $p$ with radius $\sigma^{*}(p)$ and weight it by the corresponding optimal vesselness (Eq.5).

3) Normalize each bin count on the circle so that the total count adds up to 1

4) Sort the histogram bins and save the three largest peaks and their corresponding angles.

Fig.3 illustrates circular histograms of a tubular region in (a) and junction location in (b). One can see that the eigenvectors at neighbouring points inside the vessel are oriented in the same direction. Thus, in this region, the histogram of neighbourhood orientation has only one peak. Meanwhile, at the bifurcation, where the vessel splits into two smaller vessels, there are three principal directions and the histogram has three peaks.

\section{E. Bifurcationness measure}

According to the HoE feature, a junction candidate has at least three peaks in the histogram. Thus, the proposed bifurcationness measure is set as the height of the third peak in this histogram: it will have a significant value at bifurcations only. The higher the third peak is and the stronger the bifurcationnes will be. Fig.4 shows the bifurcationness of two different images.

\section{EXPERIMENTAL RESULTS}

The method has been tested on five real clinical X-ray angiograms acquired during routine cardiac catheter examinations performed in the Cardiology Department of The University Hospital Fattouma Bourguiba, Monastir, Tunisia. The images have different levels of difficulty in terms of 

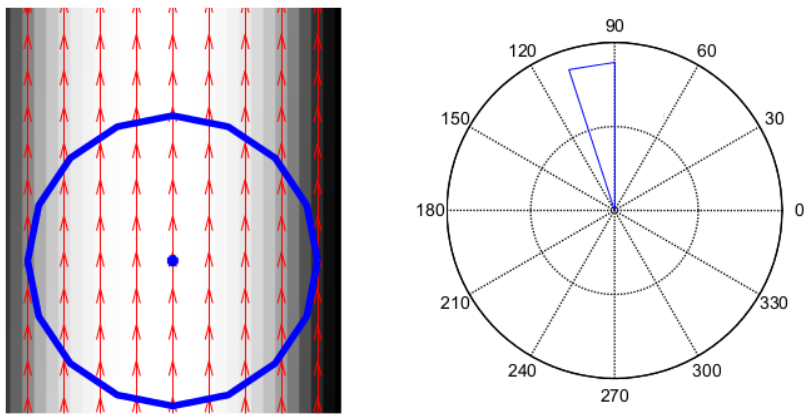

(a)
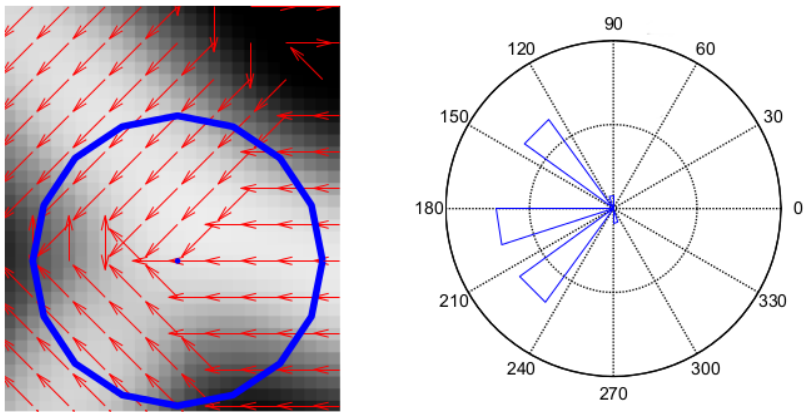

(b)

Figure 3. Illustration of Histogram of eigenvectors. (a) Eigenvector field of a tubular part and its corresponding circular Histogram. (a) Eigenvector field of a junction location and its corresponding circular Histogram.

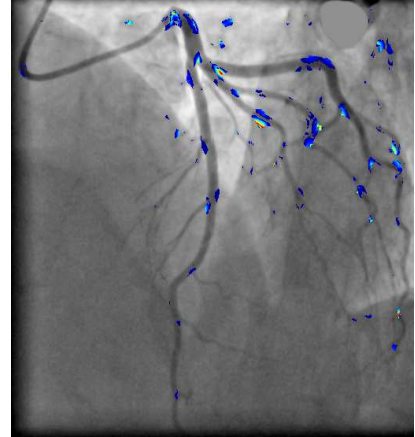

(a)

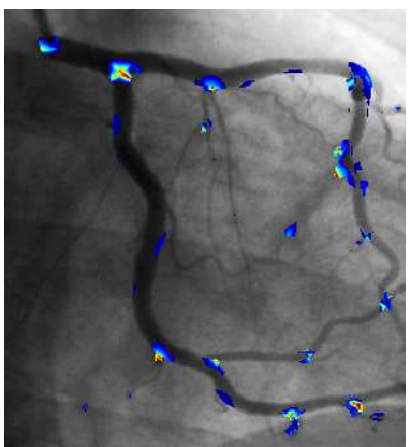

(b)
Figure 4. Bifurcationness measure superimposed to two original images.

vessel illumination and noise. All the results presented in this work use $\beta=0.5$ and half the value of the maximum Hessian norm for $\mathrm{c}\left(c=\frac{1}{2} \max \|H(x)\|_{F}\right)$ as proposed in [17].

Scales were set according to the approximate width of the smallest and largest vessel to be detected. The largest radii $\left(\sigma_{\max }\right)$ was measured and provided by the user whereas $\sigma_{\min }$ was set to 1 since vessels with smaller radii aren't of interest. Four scales between $\sigma_{\min }$ and $\sigma_{\max }$ are generated using a logarithmic progressing.
For the HoE method, only candidates with a bifurcationness measure greater than 0.1 are retained.

In terms of absolute time consuming, the Matlab code of the whole procedure on a Core i7 computer of $2.6 \mathrm{GHz}$ and $8 \mathrm{~GB}$ spends an average of 5 seconds per image

We have compared the HoE detector with the classic determinant of Hessian detector (DoH). Only pixels with DoH ratio higher than $40^{t h}$ percentile of the accumulated histogram of the $\mathrm{DOH}$ image were retained.

Both $\mathrm{HoE}$ and DoH candidates are grouped into different connected components using a labelling algorithm assuming the 8-connectivity. Then, the centre of each component is identified as the most suitable point to be centre of the junction.

The set of junction candidates is further refined through a non maximal-suppression (NMS) step based on a set of hypothesis related to the nature of images observed on our dataset. For example, the minimum distance between two junction was set to 15 pixels, thus a window of 30-by30 pixel was used for NMS and the number of points in each connected component was used as the score of the corresponding junction point. The minimum size of a retained component has been set to 5 pixels. The yellow circles in Fig.5(a) and Fig.5(b) show the final bifurcation points obtained by $\mathrm{DoH}$ while the HoE candidates are represented by the red color in Fig.5(c) and Fig.5(d).

The quantitative results of the five images for the efficiency of the junction detection process are shown in Table.I. Both DoH and HoE methods can tackle with principal bifurcations however the rate of false positives produced by $\mathrm{DoH}$ is very high (10.8 against 4.4 for HoE). The wrong detection in our method is due to background artifacts and noise. The HoE detector also gives less false negatives. The missed junctions are generally located on the crossing between very thin and noisy vessels.

\section{CONCLUSION}

In this paper, we presented a novel method for the detection of junctions in coronary X-ray angiograms. This method is based on the multiscale Hessian analysis. The Hessian eigenvectors, which are a good marker of local vessel orientation have been used to design an Histogram of Eigenvectors detector whereas its eigenvalues were used to weight this histogram and estimate the size of the junction. This leads to an adaptive HoE which behaviour is better than the classic fixed-size version. Based on this detector, the bifurcationness measure is set to the height of the third peak in the histogram. The method has been tested on real coronary artery angiographies and shows better results compared to the classic DoH detector. Overall, the proposed landmark detector approach has potential for application with other 2D and 3D vascular images.

In the future work, we would like to refine the bifurcation location and accurately detect its branches. 
Table I

COMPARISON BETWEEN DOH AND HOE IN TERMS OF NUMBER OF FALSE POSITIVES AND NUMBER OF FALSE NEGATIVES.

\begin{tabular}{|c|c|c||c|c|}
\hline \multirow{2}{*}{ Image } & \multicolumn{2}{|c||}{ DoH } & \multicolumn{2}{c|}{ HoE } \\
\cline { 2 - 5 } & FP & FN & FP & FN \\
\hline Image 1 & 6 & 5 & 4 & 3 \\
\hline Image 2 & 7 & 3 & 3 & 2 \\
\hline Image 3 & 23 & 5 & 8 & 5 \\
\hline Image 4 & 10 & 5 & 4 & 4 \\
\hline Image 5 & 8 & 5 & 3 & 2 \\
\hline mean & 10.8 & 4.6 & 4.4 & 3.2 \\
\hline
\end{tabular}

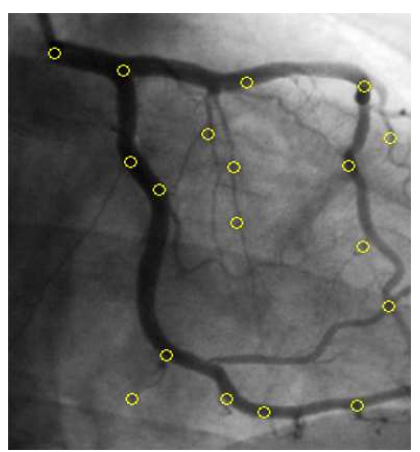

(a)

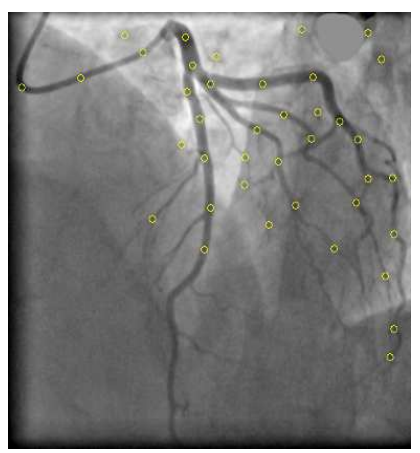

(c)

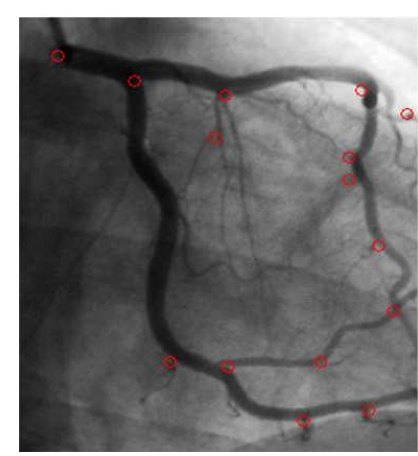

(b)

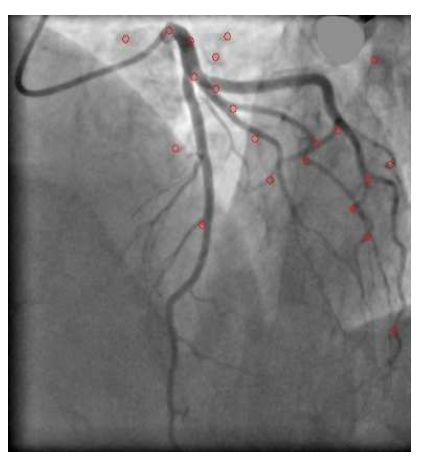

(d)
Figure 5. Comparison between DoH (yellow) and HoE (red) on two different images. The first image is Image 1 in the table. The second image (Image 3) in the table is more difficult than the first due to the low contrast and non-uniform illumination of the background.

\section{REFERENCES}

[1] J. Feng, Y.-S. Liu, and L. Gong, "Junction-aware shape descriptor for $3 \mathrm{~d}$ articulated models using local shape-radius variation," Signal Processing, vol. 112, pp. 4-16, 2015.

[2] D. Calvo, M. Ortega, M. G. Penedo, and J. Rouco, “Automatic detection and characterisation of retinal vessel tree bifurcations and crossovers in eye fundus images," Computer methods and programs in biomedicine, vol. 103, no. 1, pp. 28-38, 2011.

[3] T. A. Qureshi, A. Hunter, and B. Al-Diri, "A probabilistic model for the optimal configuration of retinal junctions using theoretically proven features," in Pattern Recognition (ICPR), 2014 22nd International Conference on. IEEE, 2014, pp. 3304-3309.

[4] Q. Lin, "Enhancement, extraction, and visualization of 3d volume data," Ph.D. dissertation, Department of Electrical Engineering, Linkoping University, SE-581 83 Linkoping, Sweden, 2003.

[5] U. T. Nguyen, A. Bhuiyan, L. Park, R. Kawasaki, T. Y. Wong, K. Ramamohanarao et al., "Automatic detection of retinal vascular landmark features for colour fundus image matching and patient longitudinal study," in Image Processing (ICIP), 2013 20th IEEE International Conference on. IEEE, 2013, pp. 616-620.

[6] R. Su, C. Sun, and T. D. Pham, "Junction detection for linear structures based on hessian, correlation and shape information," Pattern Recognition, vol. 45, no. 10, pp. 36953706, 2012.

[7] D. Baboiu and G. Hamarneh, "Vascular bifurcation detection in scale-space," in Mathematical Methods in Biomedical Image Analysis (MMBIA), 2012 IEEE Workshop on, Jan 2012, pp. 41-46.

[8] R. Su, C. Sun, C. Zhang, and T. D. Pham, "A new method for linear feature and junction enhancement in $2 \mathrm{~d}$ images based on morphological operation, oriented anisotropic gaussian function and hessian information," Pattern Recognition, no. 0, pp. -, 2014.

[9] J. M. Núñez, J. Bernal, F. J. Sánchez, and F. Vilariño, "Growing algorithm for intersection detection (graid) in branching patterns," Machine Vision and Applications, vol. 26, no. 2-3, pp. 387-400, 2015.

[10] C. Harris and M. Stephens, "A combined corner and edge detector." in Alvey vision conference, vol. 15. Citeseer, 1988, p. 50 .

[11] K. Mikolajczyk, T. Tuytelaars, C. Schmid, A. Zisserman, J. Matas, F. Schaffalitzky, T. Kadir, and L. Van Gool, "A comparison of affine region detectors," International journal of computer vision, vol. 65, no. 1-2, pp. 43-72, 2005.

[12] D. G. Lowe, "Object recognition from local scale-invariant features," in Computer vision, 1999. The proceedings of the seventh IEEE international conference on, vol. 2. Ieee, 1999, pp. 1150-1157.

[13] H. Bay, T. Tuytelaars, and L. Van Gool, "Surf: Speeded up robust features," in Computer vision-ECCV 2006. Springer, 2006, pp. 404-417.

[14] M. Zhao and G. Hamarneh, "Bifurcation detection in 3d vascular images using novel features and random forest," in Biomedical Imaging (ISBI), 2014 IEEE 11th International Symposium on, April 2014, pp. 421-424.

[15] N. Dalal and B. Triggs, "Histograms of oriented gradients for human detection," in Computer Vision and Pattern Recognition, 2005. CVPR 2005. IEEE Computer Society Conference on, vol. 1. IEEE, 2005, pp. 886-893. 
[16] T. Lindeberg, "Principles for automatic scale selection," Handbook on computer vision and applications, vol. 2, pp. 239-274, 1999.

[17] A. Frangi, W. Niessen, K. Vincken, and M. Viergever, "Multiscale vessel enhancement filtering," Medical Image Computing and Computer Assisted Interventation- MICCAI, pp. 130-137, 1998.

[18] A. Kerkeni, A. Benabdallah, and M. H. Bedoui, "Coronary artery multiscale enhancement methods: A comparative study," in Image Analysis and Recognition. Springer, 2013, pp. 510-520.

[19] A. Kerkeni, A. Benabdallah, A. Manzanera, and M. H. Bedoui, "A coronary artery segmentation method based on multiscale analysis and region growing," Computerized Medical Imaging and Graphics, vol. 48, pp. 49 - 61, 2016. 